\title{
Long-term improvement of arterial wall characteristics in patients with diabetes mellitus type 1 using cyclic, intermittent treatment with a low-dose fluvastatin and valsartan combination
}

\author{
VEDRAN SAVIĆ ${ }^{1}$, MIODRAG JANIĆ ${ }^{1}$, MOJCA LUNDER $^{1}$, KARIN KANC $^{2}$, \\ ANDREJ JANEŽ ${ }^{3}$, BARBARA ERŽEN ${ }^{1}$ and MIŠO ŠABOVIČ ${ }^{1}$ \\ ${ }^{1}$ Department of Vascular Diseases, Ljubljana University Medical Centre; \\ ${ }^{2}$ Diabetes \& Me, Private Diabetes Centre; ${ }^{3}$ Department of Endocrinology, Diabetes and Metabolic Diseases, \\ Ljubljana University Medical Centre, Ljubljana SI-1000, Slovenia
}

Received February 17, 2015; Accepted June 1, 2015

DOI: $10.3892 / \mathrm{etm} .2015 .2622$

\begin{abstract}
Improvement of arterial wall (AW) characteristics decreases cardiovascular risk. In a previous study, it was observed that AW characteristics in patients with diabetes mellitus type 1 are significantly improved by short-term treatment with a low-dose combination of fluvastatin and valsartan. Additionally, a unique phenomenon of prolonged effect after treatment discontinuation was suggested. The present study tested whether repeated treatment after a certain period results in the same beneficial effect, thereby advancing the hypothesis that cyclic treatment can provide a long-term improvement of AW characteristics. A total of 44 patients with diabetes mellitus type 1 that participated in the previous study were recruited. Six months after the discontinuation of the initial treatment, the same treatment with a low-dose fluvastatin (10 mg daily) and valsartan (20 mg daily) combination $(n=22)$ or placebo $(n=22)$ was repeated. Brachial artery flow-mediated dilation (FMD), pulse wave velocity (PWV) and carotid artery $\beta$-stiffness were measured. It was found that the beneficial effect achieved with an initial 1-month treatment was completely regained following treatment repetition: FMD improved by $50.9 \%(\mathrm{P}<0.01)$, PWV by $5.7 \%(\mathrm{P}<0.001)$ and $\beta$-stiffness by $9.9 \%(\mathrm{P}<0.001)$. In addition, a gradual decline of the obtained effects was observed, reaching the level of $9.6 \%$ for FMD, $6.3 \%$ for PWV and 9.5\% for $\beta$-stiffness 6 months after treatment discontinuation. It was observed that repetition of treatment was similarly effective as the initial intervention. The benefits achieved by treatment steadily declined with time. Combining these findings, cyclic intermittent treatment with a
\end{abstract}

Correspondence to: Professor Mišo Šabovič, Department of Vascular Diseases, Ljubljana University Medical Centre, Zaloška Cesta 7, Ljubljana SI-1000, Slovenia

E-mail: miso.sabovic@kclj.si

Key words: cardiovascular prevention, cyclic intermittent treatment, low-dose, fluvastatin and valsartan combination, diabetes mellitus type 1 , treatment repetition low-dose fluvastatin and valsartan combination is proposed as a new cardiovascular preventive strategy in patients with DM1.

\section{Introduction}

In patients with diabetes mellitus type 1, impaired functional and structural arterial wall (AW) properties, such as endothelial dysfunction and increased arterial stiffness, progressively lead to the development of cardiovascular diseases and their consequences $(1,2)$. These manifestations in patients with diabetes mellitus are accelerated and usually develop early $(3,4)$. Despite the intensive control of risk factors and strenuous treatment strategies, cardiovascular diseases continue to represent the major cause of morbidity and mortality in patients with diabetes mellitus type $1(5,6)$. Consequently, a new, effective cardiovascular preventive strategy is required. The strategy investigated in the present study is oriented towards improving AW properties per se (7). Such an approach has not previously been evaluated.

It has previously been shown that a 1-month treatment with a low-dose fluvastatin and valsartan combination is effective in improving AW properties in patients with diabetes mellitus type 1 (8) as well as in healthy middle-aged individuals (9). The beneficial effects obtained steadily declined after 1-month treatment discontinuation, although they remained significantly present after 3 months. It is well established that improvement of these AW characteristics (endothelial function and arterial stiffness) leads to cardiovascular risk improvement.

Based on the data from previous studies, it was hypothesized that a new concept for improving AW characteristics could be to take advantage of the phenomenon of prolonged efficacy after treatment discontinuation with cyclic intermittent therapy. Therefore, testing and potentially introducing this new concept was the aim of the present study.

\section{Materials and methods}

Participants and study design. The same 44 patients of both genders (25 females and 19 males) with diabetes mellitus type 1 that participated in a previous study (8) were again 
recruited in the present study, at 6 months after the discontinuation of the initial treatment. All participants were assigned to the same groups and received the same treatment as in the previous study. The treatment group $(n=22)$ received a low-dose combination of $10 \mathrm{mg}$ fluvastatin and $20 \mathrm{mg}$ valsartan daily, while the control group $(n=22)$ received a placebo. The treatment period was 30 days. Inclusion criteria were: Diagnosis of type 1 diabetes mellitus lasting $\geq 5$ years, age between 30 and 50 years, stable insulin dosage in the last 6 months, glycated hemoglobin (HbA1c) levels $<7.5 \%$ (reported in the last month) and no history of cardiovascular disease (carotid, coronary or peripheral). Exclusion criteria were smoking, and treatment with statins or any drug from the family of renin-angiotensin-aldosterone system inhibitors. The average age of participants at inclusion in the study was 35.6 \pm 2.1 years in the control group and $36.3 \pm 1.7$ years in the treatment group. The body mass index $\left(23.3 \pm 0.5 \mathrm{~kg} / \mathrm{m}^{2}\right.$ vs. $25.2 \pm 0.9 \mathrm{~kg} / \mathrm{m}^{2}$; $\mathrm{P}=0.10)$, waist-hip ratio ( $0.9 \pm 0.1$ vs. $0.9 \pm 0.1 ; \mathrm{P}=0.64), \mathrm{HbA} 1 \mathrm{c}$ values $(6.5 \pm 0.5$ vs. $6.3 \pm 0.6 ; \mathrm{P}=0.71)$ and duration of diabetes mellitus $(15.3 \pm 2.1$-years vs. $18.4 \pm 2.4$ years; $\mathrm{P}=0.35)$ did not differ between the control and treatment groups, respectively. All subjects were informed of the study protocol and gave their informed consent. Age-matched healthy individuals were also invited to participate in order to allow for basal comparison of AW characteristics in both observed groups. The National Medical Ethics Committee of Slovenia (Ljubljana, Slovenia) approved the study.

In the patients with diabetes mellitus type 1, ultrasound measurements were performed 6 months after discontinuation of the initial treatment and again at the end of the 1-month treatment repetition period; functional and structural AW characteristics were measured. Ultrasound measurements were performed at the initial visit for the age-matched individuals and the patients with diabetes mellitus type 1 . Blood pressure was measured with an automated sphygmomanometer (Welch Allyn Speidel \& Keller, Skaneateles Falls, NY, USA). Ultrasound measurements were performed under similar conditions as in the previous study, that is, in the afternoon after a 6 -h fast following their normal breakfast and morning insulin, in a quiet, temperature-controlled environment. Patients rested in a supine position for $10 \mathrm{~min}$ prior to ultrasound measurements, which were performed by a single examiner using an Aloka alfa-10 Prosound ultrasound machine with an integrated high-resolution eTracking system (Hitachi Aloka, Wallingford, CT, USA).

\section{Ultrasound measurements}

Brachial artery flow-mediated dilation (FMD) measurement. Brachial artery FMD was assessed in accordance with previously reported guidelines $(10,11)$. As previously described, an echo-machine continuously tracked and recorded the brachial artery diameter and automatically provided the FMD value. The procedure was the same as that used in the first study: The diameter of the brachial artery was continuously visualized and recorded. After monitoring its baseline diameter (1 min), the right forearm blood pressure cuff was inflated $50 \mathrm{mmHg}$ above systolic pressure (4 min), resulting in arterial occlusion. This was followed by rapid cuff deflation, which induced reactive hyperemia. The brachial artery diameter was then recorded.
Assessment of arterial stiffness parameters. Detailed measurements of arterial stiffness parameters have also already been described. They were performed on the right common carotid artery. The ultrasound device automatically determined the stiffness parameters through analysis of pulse waves. Pressure waveforms were obtained noninvasively using arterial diameter change waveforms calibrated automatically on the basis of systolic blood pressure values. Values of pulse wave velocity (PWV) and carotid artery local stiffness ( $\beta$-stiffness) were then automatically calculated by the ultrasound machine.

Statistical analysis. All values were expressed as means \pm standard error of the mean and were normally distributed. Any differences between absolute or relative values during the initial or current treatment and between the treatment and control groups were assessed through one-way analysis of variance (ANOVA). When a significant interaction was present, the Bonferroni post-test was performed. A P-value of less than 0.05 was considered to indicate a significant difference. All statistical analyses were performed using GraphPad Prism software, version 5.0 (GraphPad Inc., La Jolla, CA, USA).

\section{Results}

Patient characteristics. Patient characteristics at the beginning (day 0) and at the end (day 30) of the current treatment period are presented in Table I. No significant differences in systolic and diastolic blood pressure, total, low-density lipoprotein and high-density lipoprotein cholesterol, triglycerides and serum glucose levels were found between the control and treatment groups. Treatment with a low-dose fluvastatin and valsartan combination did not influence blood pressure, cholesterol or triglyceride values (Table I).

Comparison of the effectiveness of the current and initial treatment on the improvement of arterial wall characteristics. Six months after treatment discontinuation, participants were again invited to participate in the study. First, the baseline AW characteristics measurements were repeated, which were similar to the values at the beginning of the initial treatment. After 1-month treatment repetition with a low-dose fluvastatin and valsartan combination, all parameters describing AW characteristics improved, whereas the absolute values did not reach the level of significance (Fig. 1). Age-matched controls had FMD 4.8 $\pm 0.2 \%$; PWV 5.2 $\pm 0.1 \mathrm{~m} / \mathrm{sec}$ and $\beta$-stiffness 5.4 $\pm 0.2 \mathrm{U}$.

When the relative values (percentage of improvement after current treatment) of the current treatment were compared, it was found that all three measured AW characteristics (FMD, PWV and $\beta$-stiffness) significantly improved compared with those in the control group, as shown in Table II. No significant changes were observed in the placebo group.

Time dependency of the beneficial effects of initial and current treatments. The time dependency of the benefits of the initial and current treatments was studied. All three measured parameters improved after the initial 1-month treatment with a low-dose fluvastatin and valsartan combination. Following the discontinuation of treatment these observed benefits steadily declined in a time-dependent manner, although they continued 
Table I. Patient characteristics in the control and treatment groups at the beginning (day 0) and after 1 month of current treatment (day 30).

\begin{tabular}{|c|c|c|c|c|c|}
\hline \multirow[b]{2}{*}{ Characteristics } & \multicolumn{2}{|c|}{ Control group } & \multicolumn{2}{|c|}{ Treatment group } & \multirow[b]{2}{*}{ P-value } \\
\hline & Day 0 & Day 30 & Day 0 & Day 30 & \\
\hline Systolic BP (mmHg) & $123.0 \pm 1.8$ & $123.5 \pm 1.9$ & $119.8 \pm 2.5$ & $120.1 \pm 2.4$ & 0.43 \\
\hline Diastolic BP (mmHg) & $79.9 \pm 1.0$ & $79.2 \pm 1.0$ & $79.2 \pm 1.1$ & $78.6 \pm 1.4$ & 0.89 \\
\hline Total cholesterol (mmol/l) & $4.8 \pm 0.2$ & $4.7 \pm 0.2$ & $5.1 \pm 0.2$ & $5.0 \pm 0.2$ & 0.39 \\
\hline LDL cholesterol (mmol/l) & $2.6 \pm 0.2$ & $2.7 \pm 0.2$ & $2.6 \pm 0.1$ & $2.4 \pm 0.2$ & 0.45 \\
\hline HDL cholesterol (mmol/l) & $1.7 \pm 0.1$ & $1.7 \pm 0.1$ & $1.8 \pm 0.1$ & $1.8 \pm 0.1$ & 0.31 \\
\hline Triglycerides (mmol/l) & $1.0 \pm 0.1$ & $0.9 \pm 0.1$ & $0.8 \pm 0.1$ & $0.8 \pm 0.1$ & 0.56 \\
\hline Serum glucose (mmol/l) & $8.0 \pm 0.9$ & $7.9 \pm 0.7$ & $7.0 \pm 0.7$ & $6.3 \pm 0.7$ & 0.21 \\
\hline
\end{tabular}

The treatment group received a combination of $10 \mathrm{mg}$ fluvastatin and $20 \mathrm{mg}$ valsartan daily while the control group received placebo for 30 days. Values are expressed as mean \pm standard error of the mean ( $\mathrm{n}=22$ per group). BP, blood pressure; LDL, low-density lipoprotein; HDL, high-density lipoprotein. The P-value refers to comparison among all four measured values of separate variables.

Table II. Relative improvements (\% change) of brachial artery FMD, and common carotid artery PWV and $\beta$-stiffness values for the current treatment in the control and treatment groups.

\begin{tabular}{lcc}
\hline Variable & Control group & Treatment group \\
\hline FMD & $1.6 \pm 2.7$ & $+50.9 \pm 14.5^{\mathrm{a}}$ \\
PWV & $0.2 \pm 0.3$ & $-5.7 \pm 1.1^{\mathrm{a}}$ \\
$\beta$-stiffness & $-0.3 \pm 0.2$ & $-9.9 \pm 1.3^{\mathrm{b}}$ \\
\hline
\end{tabular}

The control group received placebo for 30 days and the treatment group received a combination of $10 \mathrm{mg}$ fluvastatin and $20 \mathrm{mg}$ valsartan daily for 30 days. Values are expressed as mean \pm standard error of the mean ( $\mathrm{n}=22$ per group). ${ }^{\mathrm{a}} \mathrm{P}<0.01$ and ${ }^{\mathrm{b}} \mathrm{P}<0.001$ for comparison with control group. FMD, flow-mediated dilation; PWV, pulse wave velocity.

to be present at the level of $66.6 \%$ for FMD, 36.1\% for PWV and $56.0 \%$ for $\beta$-stiffness 3 months after discontinuation of the initial treatment. Six months after the discontinuation of the initial treatment the observed benefits were 9.6\% for FMD, $6.3 \%$ for PWV and $9.5 \%$ for $\beta$-stiffness. At that time point, treatment was repeated (current treatment). The improvement reached levels comparable to those previously obtained with the initial treatment (Fig. 2).

\section{Discussion}

In the present study, 1-month treatment with a low-dose fluvastatin and valsartan combination was repeated 6 months following the discontinuation of the initial treatment in patients with diabetes mellitus type 1 . It was found that 6 months after initial treatment discontinuation, minimal to slight residual improvements of AW properties remained (FMD 9.6\%, PWV $6.3 \%$ and $\beta$-stiffness $9.5 \%$ ). Notably, repetition of the same treatment again led to improvement of AW properties when compared with the initial treatment (significant improvements:
A

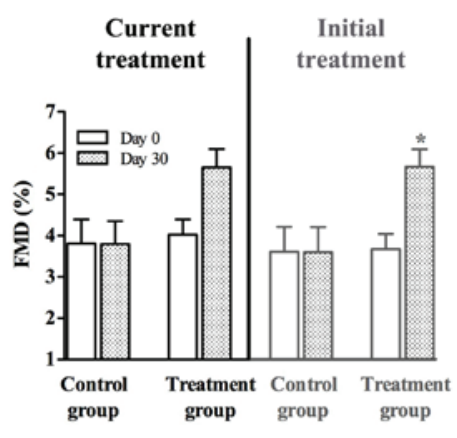

B

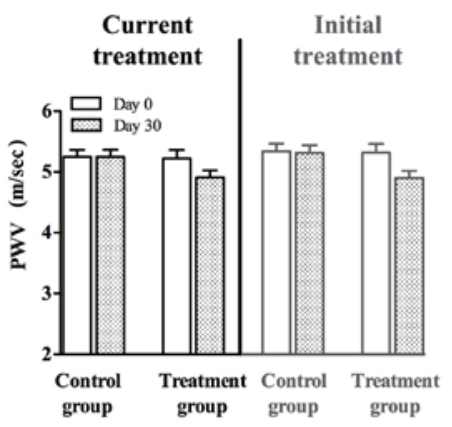

C

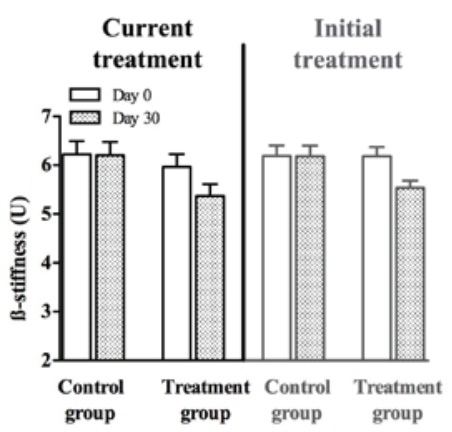

Figure 1. Values of (A) brachial artery flow-mediated dilation (FMD), and the (B) pulse wave velocity (PWV) and (C) $\beta$-stiffness of the common carotid artery at the beginning (day 0 ; white columns) and at the end (day 30 , shaded columns) of current treatment and initial treatment in the control group (received placebo for 30 days) and the treatment group (received a combination of $10 \mathrm{mg}$ fluvastatin and $20 \mathrm{mg}$ valsartan daily for 30 days). $\mathrm{P}<0.05 \mathrm{vs}$. the control group. 
A

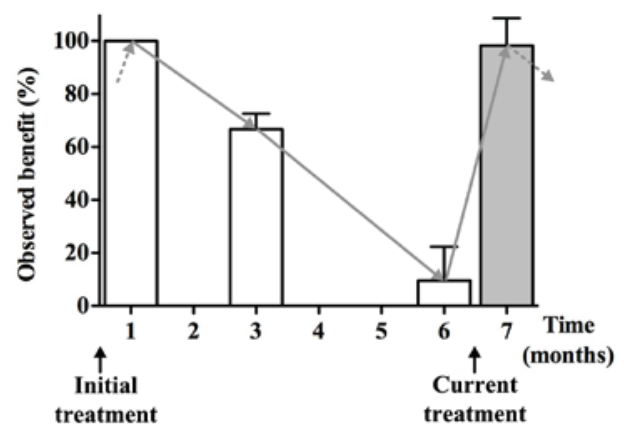

B

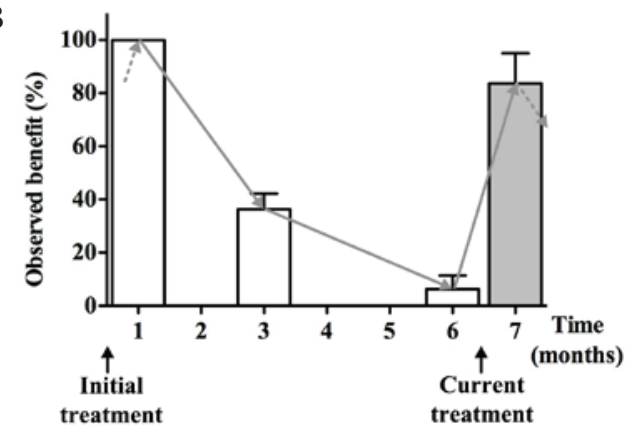

C

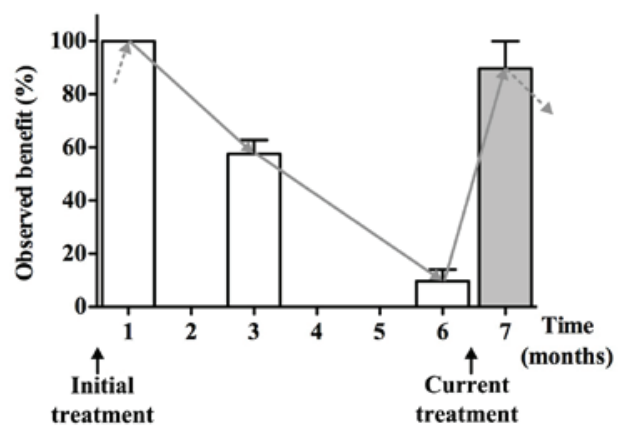

Figure 2. Time dependence of the observed benefit (\%) for (A) brachial artery flow-mediated dilation (FMD), and the (B) pulse wave velocity (PWV) and (C) $\beta$-stiffness values of the carotid artery with the initial treatment, during the rest period and with the current treatment. The treatment consisted of 1-month therapy with a low-dose fluvastatin and valsartan combination. The observed benefits were calculated with respect to the benefit of the initial treatment, which was considered as $100 \%$.

FMD $+50.9 \%$, PWV $-5.7 \%$ and $\beta$-stiffness $-9.9 \%$ ). These results strongly support a new approach to the prevention of cardiovascular disease in patients with diabetes mellitus type 1 , that is, cyclic, intermittent treatment with a low-dose fluvastatin and valsartan combination (Fig. 3).

Overall, the effectiveness of cyclic intermittent low-dose fluvastatin and valsartan combination treatment as a new approach with for the long-term improvement of AW properties in patients with diabetes mellitus type 1 , has been demonstrated. During the rest period (time without treatment) a steady but slow decline in the benefit obtained was observed. Thus, 6 months after the discontinuation of the initial treatment, only a slight improvement of AW parameters was present (FMD 9.6\%, PWV $6.3 \%$ and $\beta$-stiffness 9.5\%). At that particular time point, repetition of the same treatment (new therapeutic cycle) again lead to improvements in AW properties comparable to those of the initial treatment (significant improvements: FMD $+50.9 \%$, PWV $-5.7 \%$ and $\beta$-stiffness $-9.9 \%$ ). These results confirm the possibility of 'benefit repetition'. It is expected that the benefits

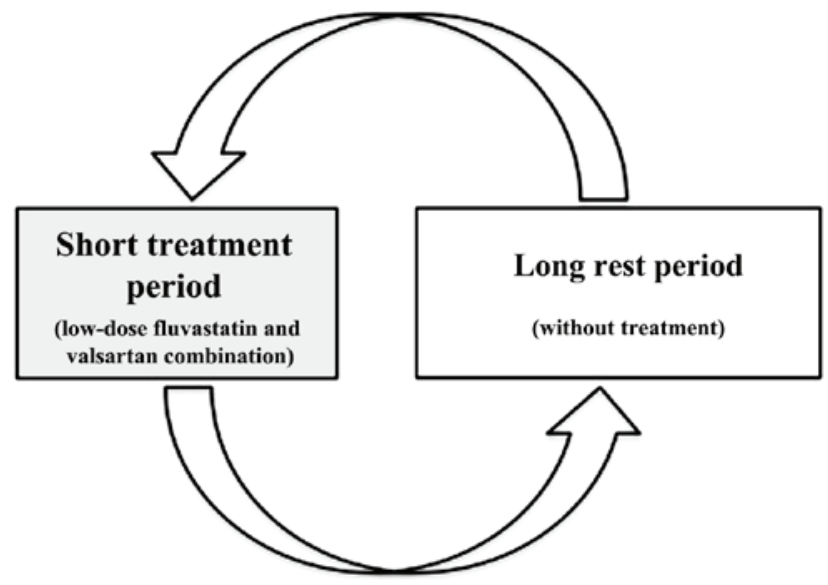

Figure 3. Schematic presentation of a new approach to cardiovascular disease prevention in patients with diabetes mellitus type 1: Cyclic, intermittent treatment with a low-dose fluvastatin and valsartan combination.

could be repeated numerous times, allowing for true long-term treatment.

When comparing the values of measured AW characteristics in patients with diabetes mellitus type 1 with those of age-matched controls, it was found, as in other studies (12-15) that patients with diabetes type 1 have impaired functional and structural AW characteristics. It seems that the changes of AW characteristics start to occur early in the disease course and slowly progress, finally leading to macrovascular complications. Taking these facts into account, it is hypothesized that patients with diabetes mellitus type 1 are ideal targets for an approach directly focusing on impaired AW properties. Long-term improvement of AW characteristics could, at least in theory but based on strong data, lead to a reduction of cardiovascular disorders and events. We consider that a cyclic, intermittent approach using sub-therapeutic dosages of well-known drugs is the most suitable, since it enables long-term improvement of AW characteristics, good compliance of the patients and no drug side-effects. Since the AW characteristics slowly declined over time (following treatment discontinuation), the identification of the period in which the benefits almost disappear was of interest. Thus, it was found that after 6 months the AW characteristics were only slightly above the initial values. It may be concluded that at this time point would be the most logical at which to investigate the possibility of effect repetition. A 6-month rest period was therefore selected.

The process of atherosclerosis is widely known to be accelerated in patients with diabetes mellitus type 1, leading to increased morbidity and mortality in this particular population (16). Furthermore, as these changes are accelerated, atherosclerotic manifestations in this particular patient group start at an earlier age. Even though there is great effort in combating risk factors for atherosclerosis in patients with diabetes mellitus type 1 , their residual cardiovascular risk remains high $(17,18)$. It should be emphasized that effects of all cardiovascular risk factors converge on the AW, which is the main focus of pathologic events in atherosclerosis as well as the main target of the present proposed approach. Furthermore, it is also known that disrupted or impaired AW properties (endothelial dysfunction and increased arterial stiffness) have a negative 
prognostic value in cardiovascular risk stratification $(19,20)$. Notably, no study or intervention has yet targeted the AW per se. Improvement of AW disorders could lead to the lowering of cardiovascular risk in patients with diabetes mellitus type 1 . To the best of our knowledge, no similar studies have been performed to date. Therefore, the present study represents a completely new cardiovascular preventive approach that has not yet been tested elsewhere.

This study leads to a proposal of a new concept: An intermittent approach comprising cyclic treatment with low-dose statin and sartan combination. The use of a low, sub-therapeutic dose of a combination of fluvastatin and valsartan is proposed. The administration of these drugs combined is proposed in a cyclical manner, with therapy repeating in certain time cycles $(6$ months in patients with diabetes mellitus type 1). Furthermore, the approach is intermittent, with therapy being administered for 1 month, followed by a so-called free-of-treatment or 'rest' period (when the beneficial effects remain present, but gradually decline). The free-of-treatment period is followed by another cycle (7). The main advantages of the approach described lie particularly in the low doses used, so there are no side-effects, while the short-term regimen improves patient compliance. Multiple repetitions, for example biannually, present repeated improvements of preclinical atherosclerosis, thus leading to postponement of its presentation in a naturally evolving process.

The major limitation of this study is that only a small group of patients was included. However, larger studies are planned in the future, as well as multiple repetition evaluation. In addition, the present study was not focused on exploring the mechanistic background of the observed beneficial effects. This is planned for larger future studies. However, it is hypothesized that the expression of vasoactive genes, and anti-inflammatory and anti-oxidative effects are at least partially involved, as shown in previous studies $(21,22)$.

In conclusion, the present study has confirmed and expanded the authors' previously established concept of low-dose intermittent treatment with fluvastatin and valsartan combination. Its effectiveness has been demonstrated in patients with diabetes mellitus type 1 and showed that after a rest period of 6 months, during which the level of benefit obtained declines, treatment repetition leads to improvements in AW properties similar to those of the initial treatment. The level of AW improvements can be again achieved after 6 months of 'rest'. These results give further support to the proposed new approach in cardiovascular disease prevention, since the combination of short 'treatment periods' and longer 'rest periods', which constitute 'cyclic treatment', would enable continuous/long-term arterial function improvement.

\section{References}

1. Davel AP, Wenceslau CF, Akamine EH, Xavier FE, Couto GK, Oliveira HT and Rossoni LV: Endothelial dysfunction in cardiovascular and endocrine-metabolic diseases: An update. Braz J Med Biol Res 44: 920-932, 2011.

2. Milan A, Tosello F, Fabbri A, Vairo A, Leone D, Chiarlo M, Covella $\mathrm{M}$ and Veglio F: Arterial stiffness: From physiology to clinical implications. High Blood Press Cardiovasc Prev 18: 1-12, 2011.

3. Christen AI, Armentano RL, Miranda A, et al: Arterial wall structure and dynamics in type 2 diabetes mellitus methodological aspects and pathophysiological findings. Curr Diabetes Rev 6 : 367-377, 2010.
4. Schram MT, Chaturvedi N, Fuller JH and Stehouwer CD; EURODIAB Prospective Complications Study Group: Pulse pressure is associated with age and cardiovascular disease in type 1 diabetes: The Eurodiab Prospective Complications Study. J Hypertens 21: 2035-2044, 2003.

5. Mangiapane H: Cardiovascular disease and diabetes. Adv Exp Med Biol 771: 219-228, 2012.

6. Sheikh-Ali M, Raheja P and Borja-Hart N: Medical management and strategies to prevent coronary artery disease in patients with type 2 diabetes mellitus. Postgrad Med 125: 17-33, 2013.

7. Janić M, Lunder M and Sabovič M: A new anti-ageing strategy focused on prevention of arterial ageing in the middle-aged population. Med Hypotheses 80: 837-840, 2013.

8. Savić V, Eržen B, Janić M, Lunder M, Boncelj M, Kanc K, Janež A and Šabović M: Improvement of arterial wall characteristics by the low-dose fluvastatin and valsartan combination in type 1 diabetes mellitus patients. Diab Vasc Dis Res 10: 420-425, 2013.

9. Lunder M, Janić M, Jug B and Sabovič M: The effects of low-dose fluvastatin and valsartan combination on arterial function: A randomized clinical trial. Eur J Intern Med 23: 261-266, 2012.

10. Corretti MC, Anderson TJ, Benjamin EJ, Celermajer D, Charbonneau F, Creager MA, Deanfield J, Drexler H, Gerhard-Herman M, Herrington D, et al: Guidelines for the ultrasound assessment of endothelial-dependent flow-mediated vasodilation of the brachial artery: A report of the International Brachial Artery Reactivity Task Force. J Am Coll Cardiol 39: 257-265, 2002.

11. Celermajer DS, Sorensen KE, Gooch VM, Spiegelhalter DJ, Miller OI, Sullivan ID, Lloyd JK and Deanfield JE: Non-invasive detection of endothelial dysfunction in children and adults at risk of atherosclerosis. Lancet 340: 1111-1115, 1992.

12. Cherney DZ and Montanari A: Gender, clamped hyperglycemia and arterial stiffness in patients with uncomplicated type 1 diabetes mellitus. Clin Exp Hypertens 36: 187-193, 2014.

13. Llauradó G, Ceperuelo-Mallafré V, Vilardell C, Simó R, Freixenet N, Vendrell J and González-Clemente JM: Arterial stiffness is increased in patients with type 1 diabetes without cardiovascular disease: A potential role of low-grade inflammation. Diabetes Care 35: 1083-1089, 2012.

14. van Elderen SG, Westenberg JJ, Brandts A, van der Meer RW, Romijn JA, Smit JW and de Roos A: Increased aortic stiffness measured by MRI in patients with type 1 diabetes mellitus and relationship to renal function. AJR Am J Roentgenol 196: 697-701, 2011.

15. Pareyn A, Allegaert K, Asscherickx W, Peirsman E, Verhamme P and Casteels K: Impaired endothelial function in female adolescents with type 1 diabetes measured by peripheral artery tonometry. Eur J Pediatr 172: 1017-1022, 2013.

16. Kanter JE and Bornfeldt KE: Inflammation and diabetes-accelerated atherosclerosis: Myeloid cell mediators. Trends Endocrinol Metab 24: 137-144, 2013.

17. Slim IB: Cardiovascular risk in type 1 diabetes mellitus. Indian J Endocrinol Metab 17 (Suppl 1): S7-S13, 2013.

18. Snell-Bergeon JK and Nadeau K: Cardiovascular disease risk in young people with type 1 diabetes. J Cardiovasc Transl Res 5: 446-462, 2012.

19. Ben-Shlomo Y, Spears M, Boustred C, May M, Anderson SG, Benjamin EJ, Boutouyrie $\mathrm{P}$, Cameron J, Chen $\mathrm{CH}$, Cruickshank JK, et al: Aortic pulse wave velocity improves cardiovascular event prediction: An individual participant meta-analysis of prospective observational data from 17,635 subjects. J Am Coll Cardiol 63: 636-646, 2014.

20. Ruggiero D, Paolillo S, Ratta GD, Mariniello A, Formisano T, Pellegrino AM and Filardi PP: Endothelial function as a marker of pre-clinical atherosclerosis: Assessment techniques and clinical implications. Monaldi Arch Chest Dis 80: 106-110, 2013.

21. Lunder M, Drevenšek G, Černe D, Marc J, Janić M and Šabovič M: Treatment with low-dose atorvastatin, losartan and their combination increases expression of vasoactive-related genes in rat aortas. J Cardiovasc Pharmacol Ther 18: 177-183, 2013.

22. Janic M, Lunder M, Prezelj M and Šabovič M: A combination of low-dose fluvastatin and valsartan decreases inflammation and oxidative stress in apparently healthy middle-aged males. J Cardiopulm Rehabil Prev 34: 208-212, 2014. 